\title{
The Triangle Law for Lyapunov Exponents of Large Random Matrices
}

\author{
Marco Isopi and Charles M. Newman* \\ Courant Institute of Mathematical Sciences, 251 Mercer Street, New York, NY 10012, USA
}

Received May 15, 1991

\begin{abstract}
For products, $A(t) \cdot A(t-1) \ldots A(1)$, of i.i.d. $N \times N$ random matrices, with i.i.d. entries, a triangle law governs the $N \rightarrow \infty$ distribution of Lyapunov exponents, much like Wigner's quarter-circle law governs the singular values of $A(1)$. Our proof requires finite fourth moments and a bounded density; the result was previously derived only in the Gaussian case.
\end{abstract}

\section{Introduction and Results}

Let $A$ be an $N \times N$ matrix whose $N^{2}$ entries are i.i.d. real random variables, distributed as $W / \sqrt{N}$, where $W$ (independent of $N$ ) has mean zero and variance one. Historically, there are three main types of results concerning the (random) singular values of $A$, i.e., the eigenvalues of $\left(A^{T} A\right)^{1 / 2}$ :

i) For $W$ Gaussian and $N$ finite, there is an explicit joint distribution of the $N$ singular values (see [Wi] and references therein).

ii) For $W$ Gaussian and $N \rightarrow \infty$, the explicit nonrandom limit $K$ of the empirical distribution function of the singular values (the "integrated density of states") is Wigner's quarter-circle law [W, p. 7]; i.e., the distribution with density

$$
K^{\prime}(\lambda)= \begin{cases}(2 / \pi)\left[1-(\lambda / 2)^{2}\right]^{1 / 2}, & 0<\lambda<2 \\ 0, & \text { elsewhere. }\end{cases}
$$

iii) $[\mathrm{MP}]$ The extension of ii) to non-Gaussian $W$ 's.

The analogues of these results for Lyapunov exponents concern products of i.i.d. matrices $A(1), A(2), \ldots$ equidistributed with $A$. The nonrandom Lyapunov exponents, $\mu_{1}^{N} \geqq \ldots \geqq \mu_{N}^{N}$, which exist by Oseledec's multiplicative ergodic theorem

* Research supported in part by NSF Grants DMS-8902156 and DMS-9196086 
$[\mathrm{O}, \mathrm{R}]$, may be defined as

$$
\mu_{k}^{N}=\lim _{t \rightarrow \infty} \frac{1}{t} \log \left(k^{\text {th }} \text { largest singular value of } A(t) \cdot A(t-1) \ldots A(1)\right) .
$$

The analogue of i), with $W$ Gaussian and $N$ finite, is an explicit expression for each of the $N$ Lyapunov exponents. This was derived first for the largest exponent in [CN] and then for all exponents in [N1]. Let $H_{N}(\lambda)$ denote the empirical distribution function of the exponentials of the $\mu_{k}^{N}$, $\mathrm{s}$ :

$$
H_{N}(\lambda)=N^{-1} \cdot\left(\text { no. of } k \text { 's in }\{1, \ldots, N\} \text { with } \exp \left(\mu_{k}^{N}\right) \leqq \lambda\right) .
$$

The analogue of ii), which follows from the explicit expressions of [N1] for the $\mu_{k}^{N}$ 's (see (2.2) below) is that for $W$ Gaussian, $H_{N}$ converges to a distribution $H$ with a triangular density function,

$$
H(\lambda)=\int_{-\infty}^{\lambda} h\left(\lambda^{\prime}\right) d \lambda^{\prime}, \quad \text { where } h(\lambda)= \begin{cases}2 \lambda, & 0<\lambda<1 \\ 0, & \text { elsewhere } .\end{cases}
$$

Equivalently, $F_{N}(\mu)$, the empirical distribution of the $\mu_{k}^{N}$, s themselves, converges to an exponential distribution,

$$
F(\mu)= \begin{cases}e^{2 \mu}, & \text { for } \mu<0 \\ 1, & \text { for } \mu \geqq 0 .\end{cases}
$$

The next theorem, which is the main result of this paper gives an analogue of iii).

Theorem 1. Assume $W$ has a finite fourth moment and a bounded density function; then as $N \rightarrow \infty, H_{N}$ converges pointwise to the triangle law distribution function $H$ and equivalently $F_{N}$ converges to the exponential distribution function $F$.

The proof of Theorem 1 is given in the next two sections. We conclude this section with two remarks.

Remark 1. In [CN] it was proved that for general $W$, the asymptotic behavior of the leading exponent is the same as for the Gaussian case (namely, $\mu_{1}^{N} \rightarrow 0$ as $N \rightarrow \infty$ ) providing that for some $\delta>0$,

$$
\left|E\left(e^{i u W}\right)\right|=O\left(|u|^{-\delta}\right) \quad \text { as } \quad|u| \rightarrow \infty .
$$

Combining the results of [Ro] (as used in Sect. 3 below) with the arguments of [CN], one can see that (1.6) may be replaced by the assumption of a bounded density. It can be shown that neither (1.6) nor the bounded density assumption implies the other. Although it is not clear what a minimal hypothesis for either $\mu_{1}^{N} \rightarrow 0$ or for $H_{N} \rightarrow H$ would look like, we note that the conclusions of Theorem 1 require the distribution of $W$ to at least be continuous. Otherwise $A$ would be rank-one with positive probability, implying $\mu_{k}^{N}=-\infty$ for $k \geqq 2$.

Remark 2. The Gaussian result of [N1] has been generalized [N2] in a different way than Theorem 1. The generalization of [N2] drops the i.i.d. entry assumption but requires, as in the Gaussian case, that the distribution of $A(1)^{T} A(1)$ be rotation invariant. The conclusion is a nonlinear mapping between two $N \rightarrow \infty$ distributions - the one for the singular values and the one for the Lyapunov exponents. Although certain technical hypotheses adopted in [N2] (see Theorem 2.11 and Remark 2.12 there) are not valid when the limiting singular value distribution is 
the quarter-circle law, nevertheless we note that the nonlinear mapping does pair the quarter-circle law with the triangle law. It is clear that some very general theorem should be valid, which would include both the results of [N2] and of this paper as special cases.

\section{Proof of Theorem 1}

Our basic approach will be to study the following limit for $0<v<1$ :

$$
G(v) \equiv \lim _{\substack{N \rightarrow \infty \\ k / N \rightarrow v}} N^{-1}\left(\mu_{1}^{N}+\ldots+\mu_{k}^{N}\right) .
$$

In the Gaussian case, one has the formula [N1]

$$
\mu_{k}^{N}=\frac{1}{2} E \log \left(\frac{Z_{1}^{2}+\ldots+Z_{N-k+1}^{2}}{N}\right),
$$

which easily implies that

$$
\lim _{\substack{N \rightarrow \infty \\ k / N \rightarrow v}} \mu_{k}^{N}=\frac{1}{2} \log (1-v) .
$$

This in turn implies both that $F_{N} \rightarrow F$ and that

$$
G(v)=\int_{0}^{v} \frac{1}{2} \log \left(1-v^{\prime}\right) d v^{\prime}=\frac{1}{2}[-v-(1-v) \log (1-v)] .
$$

Because $G$ uniquely determines $\lim F_{N}$ (see, e.g., Proposition 2.9 of [N2]), in order to prove Theorem 1, it suffices to prove that under the given hypotheses, $G(v)$ exists for each $v \in(0,1)$ and does not depend on the distribution of $W$.

We proceed along the line used in [CN] to study $\mu_{1}^{N}$. For $x_{1}, \ldots, x_{k}$ in $\mathbf{R}^{N}$, let $\left\|x_{1} \wedge \ldots \wedge x_{k}\right\|$ denote the $k$-dimensional volume of the parallelogram spanned by $x_{1}, \ldots, x_{k}$. The following lemma is a consequence of the arguments of $[\mathrm{F}, \mathrm{FK}]$; for convenience, we present a proof based on the presentation of [BL].

Lemma 1. Let $f_{1}^{N}, \ldots, f_{k}^{N}$ be orthonormal vectors in $\mathbf{R}^{N}$ and let $S_{N, k}$ denote the space of $\operatorname{such}\left(f_{1}^{N}, \ldots, f_{k}^{N}\right)$ 's. Then

$$
\mu_{1}^{N}+\ldots+\mu_{k}^{N}=\int E\left[\log \left\|A f_{1}^{N} \wedge \ldots \wedge A f_{k}^{N}\right\|\right] d \nu,
$$

where $v$ is some (unknown) probability measure on $S_{N, k}$.

Proof of Lemma 1. We apply Theorem 1.2 in Chap. 4 of [BL] (originally stated in [GR]). To verify the hypotheses of that theorem, note first that $\log ^{+}\|A\|$ is bounded by $\operatorname{tr}\left(A^{T} A\right) / 2$ so that (compare (2.14)) $E\left(\log ^{+}\|A\|\right)$ is bounded by $N / 2$, hence finite. The irreducibility and contracting hypotheses follow from Proposition 2.3 and Remark 2.4 in Chap. 4 of [BL] since the distribution of $W$ is absolutely continuous. Finally, to obtain (2.5) from the conclusions of the theorem of [BL], simply note that the action of $A$ on the $k$-fold exterior product space leaves the set of decomposable $k$-vectors (i.e., vectors of the form $x_{1} \wedge \ldots \wedge x_{k}$ ) invariant; hence the measure denoted by $v_{p}$ in $[\mathrm{BL}]$ (with $p=k$ ) is actually supported on (the projective image of) the decomposable vectors and may be replaced by some $v$ on $S_{N, k}$. 
Now (2.5) is bounded above (respectively below) by the sup (respectively inf) of the integrand over $S_{N, k}$. It immediately follows that

$$
G(v)=\lim _{\substack{N \rightarrow \infty \\ k / N \rightarrow v}} \frac{1}{N} E\left[\log \left\|A f_{1}^{N} \wedge \ldots \wedge A f_{k}^{N}\right\|\right],
$$

if this limit exists and does not depend on the choice of $\left(f_{1}^{N}, \ldots, f_{k}^{N}\right) \in S_{N, k}$. But $\left\|A f_{1}^{N} \wedge \ldots \wedge A f_{k}^{N}\right\|^{2}$ is the determinant of the $k \times k$ matrix $B$ with entries $\left(A f_{i}^{N}, A f_{j}^{N}\right)$ $=\left(f_{i}^{N}, A^{T} A f_{j}^{N}\right)$. The right-hand side of (2.6) is then $(v / 2)$ times the limit of $k^{-1} \log$ $\operatorname{det}(B)$. We wish to relate this to the limiting spectral distribution of $B$. The next lemma will help us to do so; its hypotheses were chosen for convenience and not for optimality.

Lemma 2. Let $B_{k}$ be a sequence of $k \times k$ random positive semidefinite matrices such that the (random) empirical distribution $\varrho_{k}$, of the eigenvalues of $B_{k}$, converges in probability to some (nonrandom) distribution $\varrho$ (i.e., for each continuity point $\lambda$ of $\varrho$, $\varrho_{k}(\lambda) \rightarrow \varrho(\lambda)$ in probability $)$. If

$$
\sup _{k} k^{-1} E\left(\operatorname{tr}\left(B_{k}\right)\right)<\infty
$$

and in addition the minimum eigenvalue $\lambda_{k}$ of $B_{k}$ has

$$
\text { for some } \gamma>0, \quad \sup _{k} E\left(\left(\underline{\lambda}_{k}\right)^{-\gamma}\right)<\infty,
$$

then $\int|\log \lambda| d \varrho(\lambda)<\infty$ and

$$
\lim _{k \rightarrow \infty} k^{-1} E\left[\log \left(\operatorname{det} B_{k}\right)\right]=\int_{0}^{\infty} \log \lambda d \varrho(\lambda) .
$$

Proof of Lemma 2. Let $\lambda_{i}$ denote the eigenvalues of $B_{k}$ and let $\varepsilon<1$ and $L>1$ be continuity points of $\varrho$. Then

$$
\begin{aligned}
k^{-1} \log \left(\operatorname{det} B_{k}\right)= & k^{-1} \sum_{i: \lambda_{i} \leqq \varepsilon} \log \lambda_{i}+\int_{\varepsilon+}^{L-} \log \lambda d \varrho_{k}(\lambda) \\
& +k^{-1} \sum_{i: \lambda_{i} \geqq L} \log \lambda_{i} \equiv I_{1}+I_{2}+I_{3} .
\end{aligned}
$$

Now

$$
I_{2} \rightarrow \int_{\varepsilon}^{L} \log \lambda d \varrho(\lambda)
$$

in probability, and since $I_{2}$ is bounded, $E\left(I_{2}\right)$ has the same limit. Meanwhile,

$$
\left|I_{3}\right| \leqq\left(\sup _{\lambda \geqq L} \frac{\log \lambda}{\lambda}\right) k^{-1} \operatorname{tr}\left(B_{k}\right)
$$

and

$$
\left|I_{1}\right| \leqq\left(\sup _{\lambda \leqq \varepsilon} \lambda^{\gamma}|\log \lambda|\right) \underline{\lambda}_{k}^{-\gamma}
$$

Taking expectations and then letting $L \rightarrow \infty$ and $\varepsilon \rightarrow 0$, yields the desired result by standard arguments.

To complete the proof of Theorem 1, we must show that for any choice of $\left(f_{1}^{N}, \ldots, f_{k}^{N}\right)$, the hypotheses of Lemma 2 are valid with $\varrho$ not depending on either 
the choice of $\left(f_{1}^{N}, \ldots, f_{k}^{N}\right)$ or the distribution of $W$. First we note that because the entries of $A$ are independent, mean zero, variance one random variables, we have

$$
k^{-1} E \operatorname{tr}(B)=k^{-1} \sum_{i=1}^{k} E\left\|A f_{i}^{N}\right\|^{2}=1 .
$$

This yields (2.7). Next we show convergence of $\varrho_{k}$ to a $\varrho$ not depending on $\left(f_{1}^{N}, \ldots, f_{k}^{N}\right)$ or $W$. Under our hypothesis that $E\left(W^{4}\right)<\infty$, we could obtain this from the results of $[\mathrm{MP}]$; however we will rely instead on the results of $[\mathrm{Y}]$ which do not require extra moment conditions beyond finite variance. We note that the matrix $B$ may be expressed as

$$
B=P_{k}^{T} O^{T} A^{T} A O P_{k},
$$

where $O$ is any $N \times N$ orthogonal matrix that maps the standard basis vectors $e_{1}, \ldots, e_{k}$ into $f_{1}^{N}, \ldots, f_{k}^{N}$ and $P_{k}$ is the $N \times k$ matrix with diagonal entries 1 and all others zero. The $N \times N$ matrix

$$
\widetilde{B}_{N}=A^{T} A O \widetilde{P}_{k} O^{T},
$$

where $\widetilde{P}_{k}$ is the $N \times N$ projection matrix onto the span of $e_{1}, \ldots, e_{k}$, has all the eigenvalues of $B$ plus $N-k$ extra zero eigenvalues. Thus to obtain a limiting spectral distribution for $B$, it suffices to obtain one for $\widetilde{B}_{N}$. We apply Theorem 2.1 of [Y] with the $n, p, y, X_{p}$ and $T_{p}$ of $[\mathrm{Y}]$ taken respectively as $N, N, 1, \sqrt{N} A^{T}, O \widetilde{P}_{k} O^{T}$; the quantities $H_{i}$ of $[\mathrm{Y}]$ are in our case

$$
H_{i}=\lim _{N \rightarrow \infty} N^{-1} \operatorname{Tr}\left[\left(O \widetilde{P}_{k} O^{T}\right)^{i}\right]=\lim _{N \rightarrow \infty} k / N=v \text { for all } i .
$$

The limiting spectral distribution of $\widetilde{B}_{N}$ exists and, by Eq. (4.14) of [Y], it does not depend on the distribution of $W$.

The last remaining hypothesis to verify is (2.8). This will be done in the next section. It is there that the two hypotheses on $W$ of Theorem 1 will be seriously used: bounded density in Lemmas 3 and 4; finite fourth moment in Lemma 5. (The mild use made of the existence of a density for $W$ to prove Lemma 1 was probably not necessary.)

\section{Bounds on Minimum and Maximum Eigenvalues}

Let $k=k_{N}$ be chosen so that $k_{N} / N \rightarrow v \in(0,1)$ and let $\lambda_{N}, \bar{\lambda}_{N}$ denote the minimum and maximum eigenvalues of the $k \times k$ matrix $B=B_{N}$ given by (2.15) with $O=O_{N}$ any (deterministic) sequence of $N \times N$ orthogonal matrices. To complete the proof of Theorem 1, it only remains to show that

$$
\text { for some } \gamma>0, \quad \limsup _{N \rightarrow \infty} E\left(\underline{\lambda}_{N}^{-\gamma}\right)<\infty .
$$

In order to do so, we use the following lemma which is essentially a result of [BYK]:

Lemma 3. Suppose that

$$
\text { for some } \gamma^{\prime}>0, \quad \limsup _{N \rightarrow \infty} E\left(\bar{\lambda}_{N}^{\gamma^{\prime}}\right)<\infty .
$$


Then (3.1) will be valid if for some finite $M$, every $w>0$ and every $N$,

$$
P\left(\left|\sum_{j=1}^{N} a_{j} W_{j}\right| \leqq w\right) \leqq M w \quad \text { if } \quad \sum_{j=1}^{N}\left(a_{j}\right)^{2}=1 .
$$

Here $W_{1}, W_{2}, \ldots$ are i.i.d. with the same distribution as $W$.

Proof of Lemma 3. The matrix $B_{N}$ of (2.15) may be expressed as

$$
B_{N}=\frac{1}{N} \sum_{j=1}^{N} X_{j} X_{j}^{T}
$$

where $X_{1}, X_{2}, \ldots$ are the i.i.d. $k \times 1$ columns of $\sqrt{N} P_{k}^{T} O^{T} A^{T}$. If $b=\left(b_{1}, \ldots, b_{k}\right)$ is a 1 $\times k$ unit vector, then the inner product $b X_{1}$ equals $a A_{1}$, where $a$ is the $1 \times N$ unit vector $\left(b_{1}, \ldots, b_{k}, 0, \ldots, 0\right) O^{T}$ and $A_{1}$ is the $N \times 1$ first column of $\sqrt{N} A^{T}$. Hence (3.3) implies

$$
P\left(\left|b X_{1}\right| \leqq w\right) \leqq M w \quad \text { if } \quad \sum_{j=1}^{k}\left(b_{j}\right)^{2}=1 .
$$

By the proof of Theorem 5.1 of $[\mathrm{BYK}]$, this implies that there is some constant $L<\infty$ (depending on $M$ ) such that for all large $N$,

$$
P\left(\underline{\lambda}_{N} \leqq \lambda, \bar{\lambda}_{N} \leqq R\right) \leqq L^{N}(R / \lambda)^{k / 2} \lambda^{N / 2} \text { for } 0<\lambda<R / 4 .
$$

If we set $R=\lambda^{-\theta}$ with $\theta$ chosen small enough so that $(1+\theta) v<1$ and choose $\beta<\frac{1}{2}(1-(1+\theta) v)$, then for $\lambda_{0}$ small enough, there is some $C<\infty$ so that

Thus

$$
P\left(\underline{\lambda}_{N} \leqq \lambda, \bar{\lambda}_{N} \leqq \lambda^{-\theta}\right) \leqq C \lambda^{\beta N} \text { for } 0<\lambda<\lambda_{0} .
$$

$$
P\left(\underline{\lambda}_{N} \leqq \lambda\right) \leqq C \lambda^{\beta N}+P\left(\bar{\lambda}_{N}>\lambda^{-\theta}\right) \leqq C \lambda^{\beta}+E\left(\overline{\lambda_{N}^{\gamma^{\prime}}}\right) \cdot \lambda^{\theta \gamma^{\prime}} \text { for } 0<\lambda<\lambda_{0} .
$$

Clearly (3.1) follows from (3.2) and (3.8) by standard arguments.

It remains to verify (3.2) and (3.3). Clearly (3.3) will be valid if $\sum a_{j} W_{j}$ has a density function bounded by $M$. Let us denote by $g[W]$ the density of the r.v. $W$ and by $\|g\|$ the (essential) sup norm of $g ;\|g[W]\|=\infty$ if $W$ does not have a bounded density. The next lemma yields (3.3) with $M=K^{*}\|g[W]\|=\sqrt{2}\|g[W]\|$; it is an immediate consequence of two known theorems [ $\mathrm{Ro}, \mathrm{H}]$.

Lemma 4. If $Y_{1}, Y_{2}, \ldots$ are independent r.v.'s, and $Y=\sum_{j=1}^{N} a_{j} Y_{j}$, then

$$
\|g[Y]\| \leqq K^{*}\left[\sum_{j=1}^{N}\left(a_{j}^{2} /\left\|g\left[Y_{j}\right]\right\|^{2}\right)\right]^{-1 / 2},
$$

where $K^{*}$ is a finite universal constant $(=\sqrt{2})$.

Proof of Lemma 4. The elementary fact that $\left\|g\left[X+X^{\prime}\right]\right\| \leqq\|g[X]\|$ for independent $X$ and $X^{\prime}$ shows that in Lemma 4, we may assume, without loss of generality, that $\left\|g\left[Y_{j}\right]\right\|<\infty$ for each $j$. Let $U_{1}, U_{2}, \ldots$ be i.i.d. with a uniform distribution on $(-1 / 2,1 / 2)$, so that the densities of $Y_{j}$ and $U_{j} /\left\|g\left[Y_{j}\right]\right\|$ have the same sup norm. It is proved in $[\mathrm{Ro}]$ that

$$
\|g[Y]\| \leqq g[\tilde{U}](0), \quad \text { where } \quad \tilde{U}=\sum_{j=1}^{N}\left(a_{j} /\left\|g\left[Y_{j}\right]\right\|\right) U_{j}
$$


By scaling, we easily obtain (3.9) provided only that

$$
K^{*} \equiv \sup _{N \geqq 1}\left\{\sup \left(g\left[\sum_{j=1}^{N} b_{j} U_{j}\right](0)\right)\right\}
$$

is finite, where the second sup is over all $b_{1}, \ldots, b_{N}$ with $\sum b_{j}^{2}=1$. The quantity inside the curly brackets is easily seen to be the maximum $(N-1)$-dimensional volume of slices of the unit cube in $\mathbf{R}^{N}$ by $(N-1)$-hyperplanes through its center. $K^{*}$ was proven finite in $[\mathrm{H}]$ and its value was shown to be $\sqrt{2}$ in [B].

It now only remains to verify (3.2) to finish the derivation of (3.1) and hence, by the results of Sect. 2, to complete the proof of Theorem 1. The next lemma yields (3.2) with $\gamma^{\prime}=1$; it is based on the arguments of [YBK].

Lemma 5. If $E\left(W^{4}\right)<\infty$, then for any $0 \leqq v \leqq 1$,

$$
\limsup _{N \rightarrow \infty} E\left(\bar{\lambda}_{N}\right)<\infty \text {. }
$$

Proof of Lemma 5. We first note that for fixed $N, \bar{\lambda}_{N}$ is increasing in $k$; in particular,

$$
\bar{\lambda}_{N}=\sup _{\|f\|=1}\left\|A O P_{k} f\right\|^{2} \leqq \sup _{\|f\|=1}\|A f\|^{2}=\|A\|^{2} .
$$

Hence, we restrict attention to the case $k=N$. For each $N$, we write $A=A_{N}$ as $A=A^{(1)}+A^{(2)}$, where

$$
A_{i j}^{(1)}=A_{i j} 1_{\left|A_{i j}\right| \leqq \delta_{N}}-E\left(A_{i j} 1_{\left|A_{i j}\right| \leqq \delta_{N}}\right), \quad A_{i j}^{(2)}=A_{i j}-A_{i j}^{(1)} .
$$

$\delta_{N}>0$ will be specified later, with $\delta_{N} \rightarrow 0$ as $N \rightarrow \infty$. If we define, for $m=1$ or 2 ,

$$
\bar{\lambda}_{N}^{(m)}=\left\|A^{(m)}\right\|^{2}=\text { maximum eigenvalue of }\left(A^{(m)}\right)^{T} A^{(m)},
$$

then since $\|A\| \leqq\left\|A^{(1)}\right\|+\left\|A^{(2)}\right\|$, it clearly suffices to show $\lim \sup E\left(\bar{\lambda}_{N}^{(m)}\right)<\infty$ for $m=1$ and 2 .

In Sect. 4 of [YBK], it is proved (using $E\left(W^{4}\right)<\infty$ ) that for any $\eta>4$, and any sequence of integers $l_{N}$ satisfying

$$
l_{N} / \log N \rightarrow \infty, \quad \delta_{N}^{1 / 6} l_{N} / \log N \rightarrow 0,
$$

one has for all large $N$,

$$
E\left[\left(\lambda_{N}^{(1)}\right)^{l_{N}}\right] \leqq E\left[\operatorname{tr}\left\{\left(A^{(1) T} A^{(1)}\right)^{l_{N}}\right\}\right] \leqq \eta^{l_{N}},
$$

Thus for any fixed $l^{\prime}$ (in particular for $l^{\prime}=1$ ),

$$
\limsup _{N \rightarrow \infty} E\left[\left(\bar{\lambda}_{N}^{(1)} / \eta\right)^{\prime}\right] \leqq 1+\limsup _{N \rightarrow \infty} E\left[\left(\bar{\lambda}_{N}^{(1)} / \eta\right)^{l_{N}}\right] \leqq 2 .
$$

To control $\lambda_{N}^{(2)}$, we have

$$
\begin{aligned}
E\left(\bar{\lambda}_{N}^{(2)}\right) & \leqq E\left(\operatorname{tr}\left\{A^{(2) T} A^{(2)}\right\}\right)=\sum_{i, j=1}^{N} E\left(A_{i j}^{(2) 2}\right) \\
& =N \operatorname{Var}\left(W 1_{|W|>\delta_{N} / \bar{N}} \leqq N E\left(W^{2} 1_{|W|>\delta_{N} / \bar{N}}\right) .\right.
\end{aligned}
$$

For the given distribution of $W$ with $E\left(W^{4}\right)<\infty$, we can choose a positive, nondecreasing function $h(x)$ on $[0, \infty)$ with $h(x) \rightarrow \infty$ as $x \rightarrow \infty$ such that

$$
E\left(W^{4} h(W)\right)<\infty \text {. }
$$


We then have

$$
\begin{aligned}
N E\left(W^{2} 1_{|W|>\delta_{N} \sqrt{N}}\right) & \leqq N E\left(W^{2} \cdot \frac{W^{2}}{\delta_{N}^{2} N} \cdot \frac{h(W)}{h\left(\delta_{N} \sqrt{N}\right)}\right) \\
& =\frac{1}{\delta_{N}^{2} h\left(\delta_{N} \sqrt{N}\right)} E\left(W^{4} h(W)\right) .
\end{aligned}
$$

We now choose $\delta_{N}$ tending to zero slowly enough to have

$$
\delta_{N}^{2} h\left(\delta_{N} \sqrt{N}\right) \rightarrow \infty \quad \text { as } \quad N \rightarrow \infty .
$$

Combining (3.19)-(3.22), we see that $\lim \sup E\left(\bar{\lambda}_{N}^{(2)}\right)<\infty$ (in fact $=0$ ) as desired. This completes the proof.

Acknowledgements. The authors thank D. J. Barsky, I. Goldsheid, H. Kesten, P. Laurence, J. W. Silverstein, and H.-T. Yau for useful discussions, references and suggestions.

\section{References}

[B] Ball, K.: Cube slicing in $\mathbf{R}^{n}$. Proc. Am. Math. Soc. 97, 465-473 (1986)

[BL] Bourgerol, P., Lacroix, J.: Products of random matrices with applications to Schrödinger operators. Boston: Birkhäuser 1985

[BYK] Bai, Z.D., Yin, Y.Q., Krishnaiah, P.R.: On the limiting empirical distribution function of the eigenvalues of a multivariate $F$ matrix. Theory Prob. Appl. 32, 490-500 (1987)

[CN] Cohen, J.E., Newman, C.M.: The stability of large random matrices and their products. Ann. Prob. 12, 283-310 (1984)

[F] Furstenberg, H.: Noncommuting random products. Trans. Am. Math. Soc. 108, 377-428 (1963)

[FK] Furstenberg, H., Kifer, Y.: Random matrix products and measures on projective spaces. Isr. J. Math. 46, 12-32 (1983)

[GR] Guivarc'h, Y., Raugi, A.: Frontière de Fürstenberg, propriétés de contraction et theorèmes de convergence. Zeit. Wahrscheinlichkeitstheorie Verw. Gebiete 69, 187-242 (1985)

[H] Hensley, D.: Slicing the cube in $\mathbf{R}^{n}$ and probability (bounds for the measure of a central cube slice in $\mathbf{R}^{n}$ by probability methods). Proc. Am. Math. Soc. 73, 95-100 (1979)

[MP] Marčenko, V.A., Pastur, L.A.: Distribution of eigenvalues for some sets of random matrices. Math. USSR-Sb. 1, 457--483 (1967)

[N1] Newman, C.M.: The distribution of Lyapunov exponents: exact results for random matrices. Commun. Math. Phys. 103, 121-126 (1986)

[N2] Newman, C.M.: Lyapunov exponents for some products of random matrices: exact expressions and asymptotic distributions. In: Random matrices and their applications, Cohen, J.E., Kesten, H., Newman, C.M. (eds.). Providence, RI: AMS 1986

[O] Oseledec, V.I.: A multiplicative ergodic theorem, Lyapunov characteristic numbers for dynamical systems. Trans. Mosc. Math. Soc. 19, 197-231 (1968)

[R] Raghunathan, M.S.: A proof of Oscledec's multiplicative ergodic theorm. Isr. J. Math. 32, 356-362 (1979)

[Ro] Rogozin, B.A.: Estimation of the maximum of a convolution of bounded densities. Theory Prob. Appl. 32, 48-56 (1987)

[W] Wigner, E.P.: Random matrices in physics. SIAM Rev. 9, 1-23 (1967)

[Wi] Wilks, S.S.: Mathematical statistics. New York: Wiley 1962

[Y] Yin, Y.Q.: Limiting spectral distribution for a class of random matrices. J. Multivar. Anal. 20, 50-68 (1986)

[YBK] Yin, Y.Q., Bai, Z.D., Krishnaiah, P.R.: On the limit of the largest eigenvalue of the large dimensional sample covariance matrix. Probab. Theory Relat. Fields 78, 509-521 (1988) 